\title{
EDITORIAL
}

\section{The artificial kidney induces acute kidney injury: yes}

\author{
N. Benichou', Stéphane Gaudry ${ }^{1,2,3,6^{*}}$ (D) and D. Dreyfuss ${ }^{1,4,5}$
}

(c) 2019 Springer-Verlag GmbH Germany, part of Springer Nature

Considerable numbers of lives have been saved after the invention of the artificial kidney, a revolutionary first organ-replacing extra-corporeal therapy, which participated in the development of intensive care. However, lifethreatening events such as thrombosis, infection, hemorrhage and dialytic hypotension may complicate renal replacement therapy (RRT) and may theoretically aggravate renal injury in critically ill patients with acute kidney injury (AKI). More insidiously, the RRT device itself and incomplete biocompatibility of membranes may contribute to renal injury.

By analogy with the ventilator-induced lung injury concept [1], our team recently refreshed the possibility of artificial kidney-induced kidney injury [2].

\section{From bedside ...: delayed renal recovery induced by RRT}

A classical clinical argument for RRT nephrotoxicity stems from the usually rapid decrease of residual renal function after initiation of chronic dialysis, which is more important with hemodialysis than with peritoneal dialysis [3].

Several trials have evaluated the effects of RRT modality on survival and on renal recovery. For instance, the acute renal failure trial network (ATN) study included more than 1000 patients with severe AKI and showed that intensive RRT strategies (more frequent intermittent hemodialysis or higher dose of continuous hemofiltration) did not improve mortality [4]. A post hoc analysis

\footnotetext{
*Correspondence: stephanegaudry@gmail.com

${ }^{6}$ Present Address: Intensive care unit, Hôpital Avicenne, 125 Rue de Stalingrad, 93000 Bobigny, France

Full author information is available at the end of the article
}

N. Benichou and Stéphane Gaudry contributed equally to this work. of this trial [5], concluded that more intensive modalities were associated with a greater reduction in urine output during the first 7 days of therapy. In the subgroup of hemodynamically stable patients [6] who received intermittent RRT, increasing frequency from 3 to 6 days per week was associated with impaired renal recovery at day 28. An individual patient data meta-analysis of $8 \mathrm{RCTs}$ (on both continuous and intermittent RRT) confirmed that higher RRT intensity delayed renal function recovery [7].

Contrarily to widespread opinion, CRRT does not confer a better renal prognosis compared with IHD as shown in a recent meta-analysis that included a vast majority of RCTs [8], whereas meta-analyses including mainly observational studies claimed the superiority of CRRT, a finding that likely reflects allocation bias [9].

Obviously, the least intensive RRT modality is no RRT at all in patients with no life-threatening complication of AKI, as shown in the IDEAL-ICU and AKIKI trials [10, 11]. In these two studies, a delayed RRT strategy averted the need for RRT in $38-49 \%$ of patients with severe AKI. Recovery of an adequate urine output and spontaneous creatinine decrease were observed earlier with the delayed than with the immediate RRT strategy in the AKIKI study [11]. Moreover, in the subgroup of patients with septic shock, the delayed strategy was associated with a cumulative urine output during the first 2 days that was significantly higher than with the early strategy (1881 $\mathrm{ml}$ vs $994 \mathrm{ml}, p=0.001$ ). This persisted after exclusion of patients treated with diuretics [12].

\section{To the pathology lab ...: old and recent histological observations}

Pathological examination (biopsy or autopsy) of patients receiving RRT for 3-4 weeks for ischemic AKI in Vietnam war casualties revealed focal areas of fresh (dating back $48-72 \mathrm{~h}$ only) tubular necrosis; whereas the initial

\section{实 Springer}


injury occurred several weeks ago [13]. Similar observations were recently done on an observational series of immediate post-mortem kidney autopsy in 19 septic shock patients with severe AKI [14]. Patients who had received RRT had more tubular lesions such as tubular brush border loss, necrosis and luminal cytoplasmic debris than those who had not received it (because deemed moribund).

\section{And to bench ...: mechanistical hypothesis}

The first potential mechanism involved in artificial kidney-induced kidney injury is intra-dialytic hypotension [13]. This occurred more frequently with the intensive strategy than with the less intensive strategy in the ATN study [4] because of the increased number of sessions with the former strategy. Such phenomenon may obviously be responsible for altered renal recovery. Mechanisms involved in this RRT-associated hemodynamic instability have been recently reviewed in this Journal [15]. Inter alia, lowering of cardiac output favored by ultrafiltration, osmotic and oncotic shifts, myocardial sideration and vascular resistance drop were potential mechanisms for this instability.

Biological incompatibility between patient blood and synthetic dialyzer membranes is also involved. Although the advent of so-called "biocompatible" dialysis membranes (polyacrylonitrile, polymethyl methacrylate, polysulfone) in the 1990s has allowed for less neutrophil and complement activation compared to cellulose or cuprophane membranes, hope regarding their positive effect on renal recovery has been tempered in metaanalysis [16]. Such discordant clinical data could reflect persistence of some biological incompatibility whichever the type of membrane: increased platelet activation was observed with so-called "biocompatible" hydrophobic membranes (polysulfone, polymethyl methacrylate), leading to platelets-neutrophils micro-aggregate formation that produced oxidative stress [17]. So hoping that blood-membranes interactions no longer exist and that RRT is safe on this point of view is probably illusory: a polysulfone is not an endothelium.

Catheter-related complications (hemorrhage, infections) may also participate in the scenario [18]. Of note,

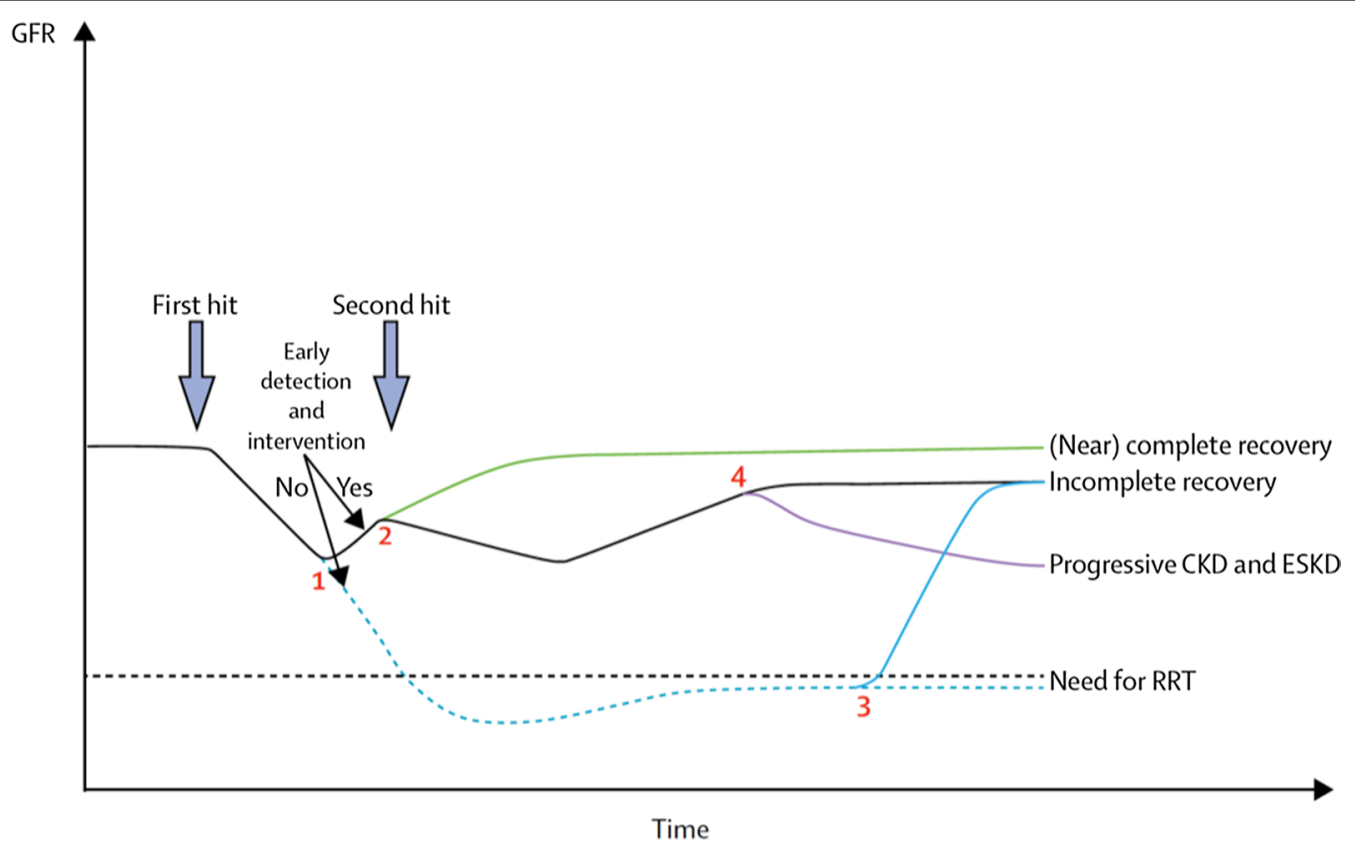

Fig. 1 Course of acute kidney injury over time. (1) Preventive action can be taken when acute kidney injury (AKI) is discovered at an early stage, and progression to the need for renal replacement therapy (RRT; dotted blue line) can potentially be avoided (full black line). (2) During recovery from AKI, the kidneys are more susceptible to further injury, which can result in new deterioration of renal function (full black line) rather than recovery (green line). (3) Patients can recover their kidney function after starting RRT (full blue line). This recovery is often incomplete, which can result in progressive chronic kidney disease (CKD) and eventually end-stage kidney disease (ESKD; full lilac line). (4) Patients who have had a second AKI hit rarely recover their kidney function completely (full blue line), and have an increased risk of progressive CKD and evolution to ESKD over time (full lilac line). The concept of artificial kidney-induced kidney injury would imply that RRT might constitute this second hit. GFR glomerular filtration rate Reprinted from The Lancet, vol 389, Vanmassenhove J, Kielstein J, Jörres A, Biesen WV, Management of patients at risk of acute kidney injury, pages 2139-2151, copyright 2017, with permission from Elsevier 
such infections were more frequent with an early RRT initiation strategy [11].

Another hypothetical mechanism was recently raised: AKI is responsible for Nicotinamide adenine dinucleotide $(\mathrm{NAD}+)$ depletion which impedes tubular regeneration [19]. This is all the more important, since RRT may remove circulating niacin, a precursor of NAD + with a molecular weight close to creatinine [2] further compromising renal recovery.

The importance of a "second hit" (infection, shock, toxic drugs) on a repairing kidney was recently emphasized [20] (see Fig. 1). Whichever the exact underlying mechanisms involved, RRT might constitute such "second AKI hit" that applied on an injured tubular epithelium sensitized to a harmful environment may lead to maladaptive repair.

In conclusion, the artificial kidney-induced kidney injury hypothesis is supported by mounting evidence. It might result in a real paradigm shift, as had been the emergence of the concept of ventilator-induced lung injury [1] for ARDS management 20 years ago.

After decades of "interventions race", the old adage "Primum non nocere" would ensure current direction of intensive care in several areas: less ventilation, less sedation, less transfusion, less antibiotics, and now less RRT unless really needed.

\begin{abstract}
Author details
${ }^{1}$ French National Institute of Health and Medical Research (INSERM), UMR S1155, Remodeling and Repair of Renal Tissue, Hôpital Tenon, Sorbonne Université, 75020 Paris, France. ${ }^{2}$ Service de Réanimation Médico-Chirurgicale, APHP, Hôpital Avicenne, 125 Rue de Stalingrad, 93000 Bobigny, France. ${ }^{3}$ Health Care Simulation Center, UFR SMBH, Université Paris 13, Sorbonne Paris Cité, Bobigny, France. ${ }^{4}$ Service de Réanimation Médico-Chirurgicale, AP-HP, Hôpital Louis Mourier, 178 rue des Renouillers, 92700 Colombes, France. ${ }^{5}$ Université de Paris, Paris, France. ${ }^{6}$ Present Address: Intensive care unit, Hôpital Avicenne, 125 Rue de Stalingrad, 93000 Bobigny, France.
\end{abstract}

\section{Compliance with ethical standards}

\section{Conflicts of interest}

Nicolas Benichou is the recipient of a grant from the Société Francophone de Néphrologie, Dialyse et Transplantation, outside of the submitted work. Stéphane Gaudry reports grants from French ministry of health, during the conduct of AKIKI trial. Didier Dreyfuss reports grants from French ministry of health, during the conduct of AKIKI trial.

\section{Publisher's Note}

Springer Nature remains neutral with regard to jurisdictional claims in published maps and institutional affiliations.

Received: 15 October 2019 Accepted: 1 December 2019

Published online: 12 December 2019
References

1. Dreyfuss D, Saumon G (1998) Ventilator-induced Lung Injury. Am J Respir Crit Care Med 157:294-323

2. Gaudry S, Quenot J-P, Hertig A et al (2019) Timing of renal replacement therapy for severe acute kidney injury in critically III patients. Am J Respir Crit Care Med 199:1066-1075

3. Moist LM, Port FK, Orzol SM et al (2000) Predictors of loss of residual renal function among new dialysis patients. J Am Soc Nephrol 11:556-564

4. VA, NIH Acute Renal Failure Trial Network, Palevsky PMZJ, O'Connor TZ et al (2008) Intensity of renal support in critically ill patients with acute kidney injury. N Engl J Med 359:7-20

5. Mc Causland FR, Asafu-Adjei J, Betensky RA et al (2016) Comparison of urine output among patients treated with more intensive versus less. intensive RRT: results from the acute renal failure trial network study. Clin J Am Soc Nephrol 11:1335-1342

6. Vijayan A, Delos Santos RB, Li T et al (2018) Effect of frequent dialysis on renal recovery: results from the acute renal failure trial network study. Kidney Int Rep 3:456-463

7. Wang Y, Gallagher M, Li Q et al (2018) Renal replacement therapy intensity for acute kidney injury and recovery to dialysis independence: a systematic review and individual patient data meta-analysis. Nephrol Dial Transpl 33:1017-1024

8. Nash DM, Przech S, Wald R, O'Reilly D (2017) Systematic review and metaanalysis of renal replacement therapy modalities for acute kidney injury in the intensive care unit. J Crit Care 41:138-144

9. Schneider AG, Bellomo R, Bagshaw SM et al (2013) Choice of renal replacement therapy modality and dialysis dependence after acute kidney injury: a systematic review and meta-analysis. Intensive Care Med 39:987-997

10. Barbar SD, Clere-Jehl R, Bourredjem A et al (2018) Timing of renal-replacement therapy in patients with acute kidney injury and sepsis. N Engl J Med 379:1431-1442

11. Gaudry S, Hajage D, Schortgen F et al (2016) Initiation strategies for renal-replacement therapy in the intensive care unit. N Engl J Med 375:122-133

12. Gaudry S, Hajage D, Schortgen F et al (2018) Timing of renal support and outcome of septic shock and acute respiratory distress syndrome. a post hoc analysis of the AKIKI randomized clinical trial. Am J Respir Crit Care Med 198:58-66

13. Conger JD (1990) Does hemodialysis delay recovery from acute renal failure? Semin Dial 3:146-148

14. Lerolle N, Nochy D, Guérot E et al (2010) Histopathology of septic shock induced acute kidney injury: apoptosis and leukocytic infiltration. Intensive Care Med 36:471-478

15. Douvris A, Zeid K, Hiremath S et al (2019) Mechanisms for hemodynamic instability related to renal replacement therapy: a narrative review. Intensive Care Med 45:1333

16. Alonso A, Lau J, Jaber BL (2008) Biocompatible hemodialysis membranes for acute renal failure. Cochrane Database Syst Rev. https://doi. org/10.1002/14651858.CD005283.pub2

17. Itoh S, Susuki C, Tsuji T (2006) Platelet activation through interaction with hemodialysis membranes induces neutrophils to produce reactive oxygen species. J Biomed Mater Res A 77:294-303

18. Buetti N, Ruckly S, Lucet J-C et al (2019) Short-term dialysis catheter versus central venous catheter infections in ICU patients: a post hoc analysis of individual data of 4 multi-centric randomized trials. Intensive Care Med 45(12):1774-1782

19. Poyan Mehr A, Tran MT, Ralto KM et al (2018) De novo NAD + biosynthetic impairment in acute kidney injury in humans. Nat Med 24:1351-1359

20. Vanmassenhove J, Kielstein J, Jörres A, Biesen WV (2017) Management of patients at risk of acute kidney injury. Lancet 389:2139-2151 